\title{
Registros de mayor altitud para mosquitos (Diptera: Culicidae) en Venezuela
}

\author{
Juan-Carlos Navarro $^{1 *}$, Fabiola Del Ventura ${ }^{1}$, Adriana Zorrilla ${ }^{1} \&$ Jonathan Liria ${ }^{2}$ \\ 1. Instituto de Zoología Tropical, Laboratorio Biología de Vectores, Universidad Central de Venezuela, Apdo. 47058, \\ Caracas 1041-A, Venezuela; juan.navarro@ciens.ucv.ve, delventurafabiola@gmail.com, adrianazg8@yahoo.es \\ 2. Departamento de Biología, Facyt. Universidad de Carabobo, Valencia, Edo. Carabobo. Venezuela; \\ jonathan.liria@gmail.com \\ * Autor de Correspondencia
}

Recibido 19-V-2009. Corregido 17-VIII-2009. Aceptado 17-IX-2009.

\begin{abstract}
Highest mosquito records (Diptera: Culicidae) in Venezuela. Mosquitoes (Diptera: Culicidae) are holometabolous insects with aquatic immature stages, which use a broad variety of larval habitats, from ground water bodies to Phytothelmata (water deposits in plants) and artificial deposits. The availability of breeding sites often determines the upper limits of mosquito ranges. We built a database with 9607 records with 432 localities, 19 genera and 254 species. The Andean mountains have $77 \%$ of the highest mosquito records including Aedes euris with record at $3133 \mathrm{~m}$, followed by three species of Anopheles -subgenera Kerteszia- with the upper limit of $2680 \mathrm{~m}$. Wyeomyia bicornis and Culex daumastocampa at $2550 \mathrm{~m}$ were the highest records in the CentralCoastal cordillera, while the highest record in Pantepui was Wyeomyia zinzala at $2252 \mathrm{~m}$. The species associated with phytothelmata (Bromeliaceae and Sarraceniaceae) represent $60 \%$ of the records. The upper limits of Culex quinquefasciatus and Anopheles (Kerteszia) species could represent the theoretical limit for transmission of filariasis or arboviruses, by Culex, and malaria by Anopheles (Kerteszia) in Venezuela. Similarly, a vector of Dengue, Aedes aegypti, has not been not recorded above 2000 m. Rev. Biol. Trop. 58 (1): 245-254. Epub 2010 March 01.
\end{abstract}

Key words: Aedes, Anopheles, Bromeliaceae, Culex, Diptera, diversity, Heliamphora, Wyeomyia, phytothelmata.

La familia Culicidae (Diptera: Nematocera) incluye cerca de 3507 especies en el mundo (WRBU 2009), con una parte importante de ellas como insectos vectores de diferentes patógenos (arbovirus, protozoos, nemátodos) que producen variadas enfermedades de importancia en salud pública y veterinaria. En Venezuela, se estiman cerca de 315 especies (Sutil 1980, Navarro et al. 2007), incluidas en 23 géneros. Estas especies utilizan una diversidad de ambientes de cría desde cuerpos de agua en el suelo, lagunas, lagos, desbordes de ríos, plantas que poseen estructuras que almacenan agua o Phytothelmata (del griego Phyto= planta y Thelmos (recipiente), en español Fitotelmata: Maguire 1971, Fish 1983, Frank 1983, Navarro et al. 2007), así como recipientes artificiales antropogénicos.

Diversos trabajos se han realizado en Venezuela, orientados hacia la historia natural de la familia Culicidae, así como estudios taxonómicos y eco-epidemiológicos en malaria y diversos arbovirus, sin embargo, pocos estudios de distribución geográfica han sido recopilados desde las contribuciones clásicas de CovaGarcía (1951) y Cova-García et al. (1966), Sutil (1980) y Heinemann \& Belkin (1978), las cuales tampoco incluyen variables ecológicas y/o biogeográficas. Los estudios más recientes son el de Rubio-Palis \& Zimmermann (1997) con vectores de malaria (Anophelinae) y su distribución por eco-regiones en el Neotrópico; 
en Venezuela, el de Navarro et al. (2007) con mosquitos asociados a fitotelmata en áreas protegidas mediante análisis biogeográficos explícitos (cladísticos) y el análisis biogeográfico más completo (no publicado, Del Ventura 2008) del cual extraemos los datos concernientes a los registros de mayor altitud mostrados aquí, debido a la ausencia de una revisión relacionada con el factor altitud en la distribución geográfica de los Culicidae de Venezuela.

El conocimiento de la distribución geográfica de estas especies es importante para el estudio de su historial natural, bionomía, ecología, genética y evolución, así como para obtener información necesaria para entender los factores biogeográficos e históricos de las diferentes enfermedades y desarrollar estrategias eficientes de control de vectores y de las enfermedades asociadas.

Una de las variables más importantes a estudiar es la distribución altitudinal de estas especies, la cual está determinada por factores intrínsecos a su biología, asociada entre otras, a los límites verticales de los sitios de cría. La altitud, la pendiente, suelos, temperatura promedio y climatología general, determinan la vegetación predominante y éstos a su vez los criaderos disponibles.

Una revisión de las zonas altas en los sistemas montañosos de Venezuela asociada con la construcción de una base de datos con información bibliográfica, de museos y/o colecciones de los mosquitos de Venezuela, nos llevó a determinar los registros de mosquitos con las mayores altitudes en el país y sus implicaciones, lo cual se muestra en el presente trabajo.

En estos sistemas montañosos se registran las especies de mosquitos de mayor altitud en Venezuela (por encima de los $2000 \mathrm{~m}$ ). Mayores esfuerzos de muestreo en zonas altas montañosas del país serán necesarios para fortalecer la información base que se presenta aquí, la cual proviene de los estudios de diversidad realizados en mosquitos, desde pioneros como Romero-Sierra, Anduze, Gabaldón, Cova-García, Belkin y sus colaboradores hasta los trabajos más recientes.
Un factor importante en la fauna de culícidos, lo representan aquellas especies asociadas a fitotelmata, por ser éstos criaderos naturales y de gran alcance altitudinal. Casi tres décadas de trabajo han transcurrido en Venezuela dedicados al inventario de la fauna de mosquitos (Diptera: Culicidae) asociados a fitotelmatas, desde los primeros trabajos realizados por Seifert (1980), Seifert \& Barrera (1981), Lounibos \& Machado-Allison (1983), Machado-Allison et al. $(1985,1986)$, luego de un inventario previo realizado en el Proyecto "Mosquitoes of Middle America" liderado por John Belkin y colaboradores en los años 70's (Heinemann \& Belkin 1978), el cual no estuvo directamente relacionado con mosquitos en este tipo de criaderos. Estos trabajos, sumados a otros sobre aspectos ecológicos de los mosquitos en fitotelmata (Barrera et al., 1989; Barrera \& Villalba, 1994) y un proyecto dedicado al binomio mosquito-fitotelmata (Navarro 1996a, 1996b, 1998, Navarro et al. 1994, Harbach \& Navarro 1996, 1995, Navarro \& Liria 2000, Liria \& Navarro 2003), cuyos resultados finales mostraron el inventario faunístico bajo una perspectiva biogeográfica histórica con métodos cladísticos antes referido (Navarro et al. 2007), forman la base de datos que se elaboró, incluyendo además los registros de la literatura existente hasta 2008 y de los museos nacionales, así como los registros de capturas de adultos (Del Ventura 2008).

\section{MATERIALES Y MÉTODOS}

Área de estudio: Venezuela esta localizada entre los meridianos $59^{\circ} 48^{\prime}$ y el $73^{\circ} 25^{\prime} \mathrm{W}$. Latitudinalmente, al norte del Ecuador, entre los paralelos $10^{\circ} 38^{\prime} 53^{\prime \prime}$ y $12^{\circ} 12^{\prime} \mathrm{N}$.

Sistemas montañosos y altitudes máximas en Venezuela: Las zonas altas montañosas de Venezuela se encuentran en las principales cordilleras, La Cordillera Andina-Sierra de Perijá, la Cordillera Central o Cordillera de la Costa en toda su extensión norte y en el área de Pantepui en los estados Bolívar y Amazonas. 
En el norte montañoso, se diferencian dos sistemas: Andino y de la Costa. La región de los Andes orientales se divide en: 1) cordillera de Perijá, que sigue en dirección N-NE y se prolonga en la península Guajira, 2) Altos páramos, de $5000 \mathrm{~m}$ de altitud, que descienden hacia las mesetas de Barquisimeto (400-1 000 m) y Coro (1000-1900 m) y 3) Cordillera de Mérida (Cárdenas 1965, Eguía 1991).

La cordillera de Mérida en los Andes, se divide en las sierras: Nevada, Santo Domingo y del Norte. La Sierra Nevada alcanza la altura máxima en el pico Bolívar (5 007 m), la Sierra de Santo Domingo, culmina en el pico Mucuñuque (4 $672 \mathrm{~m}$ ) y la sierra del Norte o de la Culata, tiene su máxima altura en Piedras Blancas (4 762 m) (Schubert 1982). El sistema cordillera de la costa o antillano, tiene su máxima altura en el pico Naiguatá (2 765 m) (González de Juana 1938, Eguía 1991).

En el S-SE del país, el Macizo GuayanésPantepui está constituido por mesetas (tepui) de areniscas, que culminan en el monte Roraima (2 $810 \mathrm{~m}$ ), donde termina la Guayana venezolana por el sureste. De Norte a Sur se encuentran sierras y tepuis cuyas máximas alturas se alcanzan en Marahuaca (2 579 m), Yaví (2 441 m) y Duida (2 400 m) (Eguía 1991).

Datos utilizados: Los registros provienen de la revisión de colecciones de mosquitos del Museo Entomológico "Doctor Pablo Cova García" del Instituto de Altos Estudios Superiores en Salud Pública Dr. Arnoldo Gabaldón, Maracay (Knight \& Stone 1977, Guimaraes 1997) y el Laboratorio de Biología de Vectores, Museo de Biología-UCV LBV (Guimaraes 1997), las dos colecciones institucionales de Culicidae más importantes del país. Adicionalmente, se usaron los datos existentes en la literatura: Mosquitos de Meso-América Heinemann \& Belkin (1978), Enumeración histórica y geográfica de las especies de Culicidae de Venezuela ordenadas según su taxonomía (Sutil 1980), Distribución geográfica y datos bionómicos de los Anofelinos de Venezuela (Cova-García 1951), Systematic Database of Diptera of the Americas South of the United States (Family CULICIDAE) (Guimaraes
1997), Contributions of the American Entomological Institute Vol. 2, 3, 4, 7, 13, 17 y 27 y los datos de la página electrónica de WRBU (2009) http://www.mosquitocatalog.org/, con la finalidad de agrupar la mayor cantidad de datos existente de Venezuela.

Igualmente, se incluyó el material recolectado en fitotelmata en una reciente exploración a la zona más alta de la Cordillera de la Costa, Parque Nacional El Ávila, registros que por su altitud máxima al norte del país indujo a la formulación de este trabajo con la inclusión de los registros totales del país. En esta localidad se recolectaron ejemplares en una transecta altitudinal desde los $1000 \mathrm{~m}$ en la ciudad de Caracas, hasta el Pico Naiguatá a $2765 \mathrm{~m}$, punto más alto de la Cordillera de la Costa. Estas larvas de mosquitos se encontraron en el límite superior de la distribución de las fitotelmatas en este Parque, entre las altitudes de 2 402-2 $537 \mathrm{~m}$, en el sendero que va desde el Pico Goering 2440 m hasta el Pico Naiguatá $2765 \mathrm{~m}$, localizado en las coordenadas $\left(10^{\circ} 32^{\prime} 6.4^{\prime \prime} \mathrm{N}-66^{\circ} 48^{\prime} 12.2^{\prime}\right.$ " W, y $10^{\circ} 32^{\prime} 24.1^{\prime \prime} \mathrm{N}-66^{\circ} 47^{\prime} 59.2^{\prime \prime}$ W). La metodología de muestreo, cría e identificación fue la referida en Navarro et al. (2007) y Navarro \& Liria (2007).

La base de datos se realizó en Excel (Microsoft Office ${ }^{\circledR}$ ), con las coordenadas geográficas de los sitios de colecta (expresadas en el sistema sexagesimal), nombre de la localidad, estado y nombre de las especies, denotando presencia o ausencia de las mismas en dichas localidades. Esta estructura de base de datos es compatible con los programas de edición de mapas y permite la posibilidad de modificar y/o agregar nueva información. Se realizaron ajustes a los datos debido a la falta de uniformidad de la información contenida tanto en las colecciones como en la literatura (carencia de datos). La georeferenciación se realizó mediante Gacetillas Geográficas de Venezuela del Instituto Geográfico de Venezuela Simón Bolívar, localizaciones por GPS (obtenidas en campo por los recolectores) y el Portal de Global Biodiversity Information Facility (http://data.gbif.org./). 


\section{RESULTADOS}

La base de datos resultó en un total de 9 607 registros, 432 localidades, 19 géneros y 254 especies debidamente georeferenciadas con ejemplares de museo y/o información específica de la literatura (Del Ventura 2008) de la cual se muestra aquí los registros de mayor altitud de zonas montañosas por encima de $2000 \mathrm{~m}$.

La Cuadro 1 y Fig. 1 muestran las especies de Culicidae, las localidades, la altitud y el perfil de alturas por regiones. Estas especies representan los registros con mayor altitud en Venezuela, tanto para mosquitos cuyas fases inmaduras se desarrollan en fitotelmatas así como en otros criaderos, con detalles de coordenadas, entidad política y referencia de su registro.

De las trece localidades de altitud mayor a $2000 \mathrm{~m}$ con registros de mosquitos, diez (77\%) pertenecen al sistema de la cordillera de Los Andes (localidades 1, 2, 3, 4, 5, 6, 8, 9, 11, 12), dos de ellas pertenecen al sistema de Cordillera Central de la Costa (15.3\%, loc. 10 y 13), y solo al Macizo Guayanés (Pantepui) (7.7\%, loc. 7). Por otra parte, se observa como en ocho de las trece localidades (61\%) están presentes especies asociadas a fitotelmata (subgéneros Microculex de Culex, Nunezia de Wyeomyia, Kerteszia de Anopheles y el género Runchomyia, en las localidades 1, 5, 6, 7, 8, 10, 11, 13 , mientras que el $39 \%$ de estas localidades (2, $3,4,9,12$ y 13) lo representan especies que se

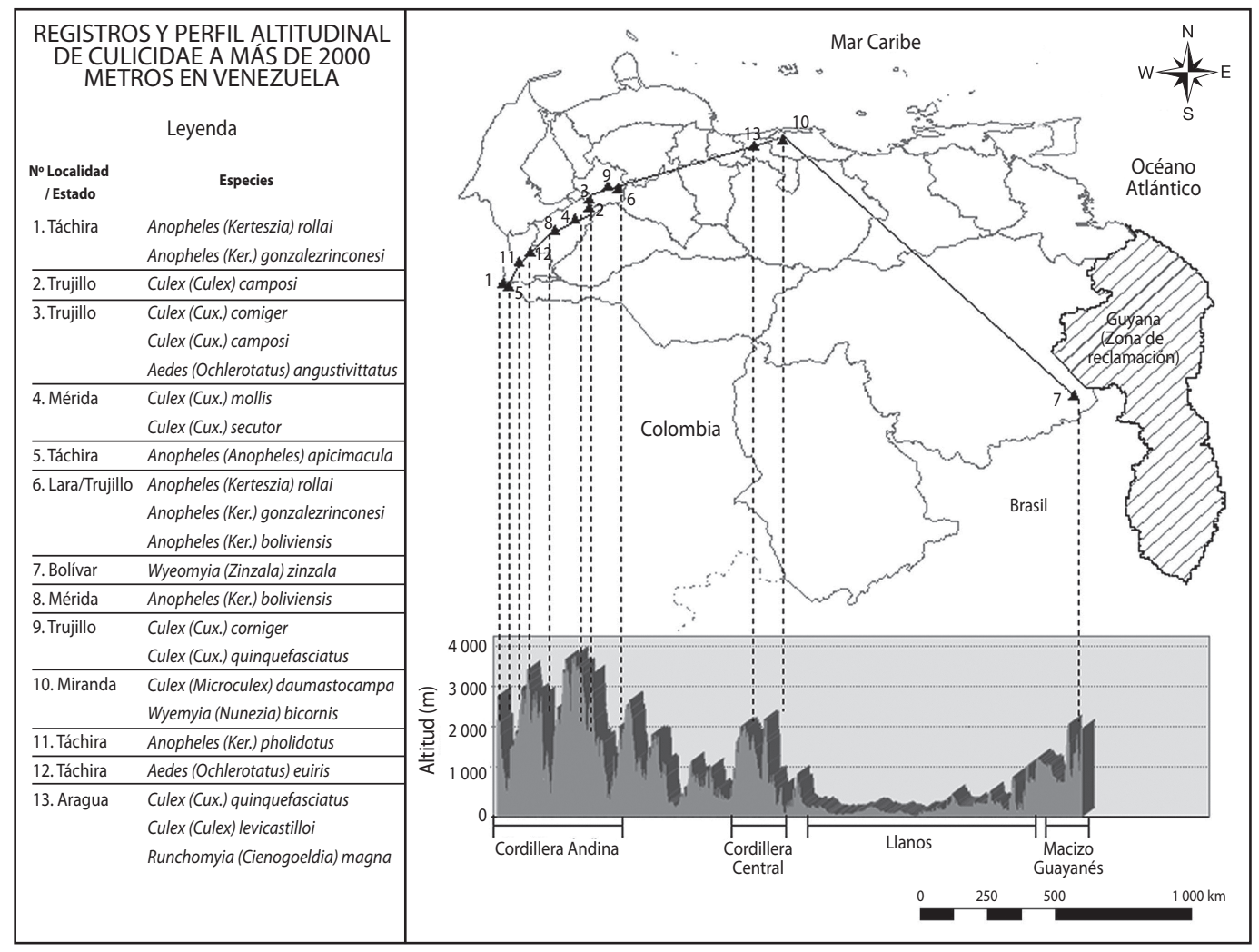

Fig. 1. Registros de mosquitos en localidades por encima de $2000 \mathrm{~m}$ en Venezuela y perfil de altura por regiones en sentido Este-Oeste.

Fig. 1. Records of mosquitoes in locations above $2000 \mathrm{~m}$ in Venezuela and the graphic altitude profile by regions in EastWest direction. 


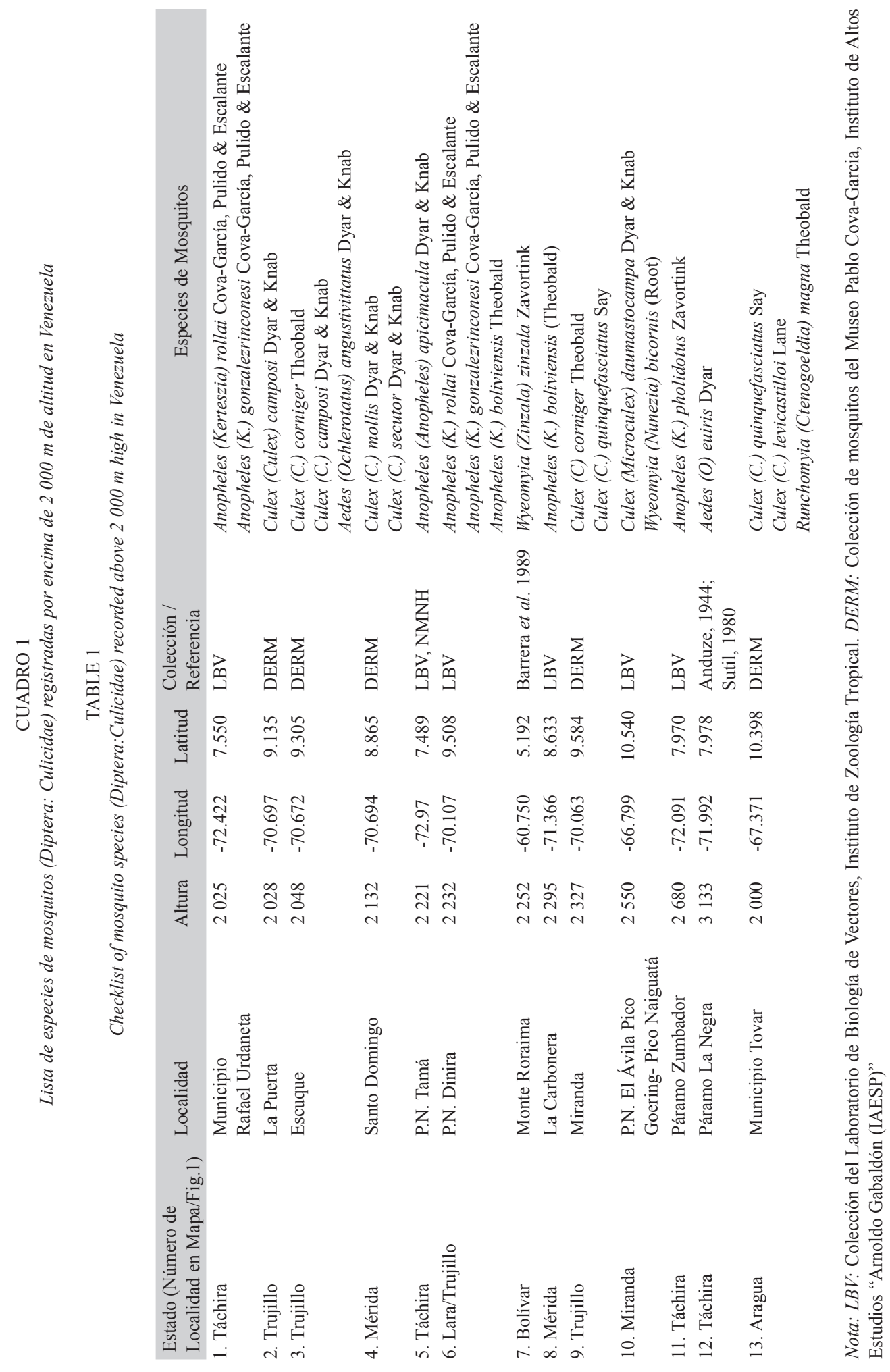


desarrollan en otro tipo de ambiente (charcas, recipientes artificiales, etc.).

La máxima altitud registrada resultó para una especie no-fitotélmica como Aedes euris Dyar, a 3133 m en Los Andes, localidad 13, seguida de Anopheles pholidotus Zavortink en Páramos de Zumbador en el estado Táchira a 2680 m. Otras tres especies de Kerteszia, tienen registros en localidades de gran altitud en los Andes: A. gonzalezrinconesi Cova-García, Pulido \& Escalante, A. rollai Cova-García, Pulido \& Escalante y A. boliviensis (Theobald), las cuales han sido muestreadas en el Parque Nacional Dinira a 232 m (Prado 2003, Navarro et al. 2007).

En un segundo orden de altitud se encuentran las especies Wyeomyia (Nunezia) bicornis (Root) y Culex (Microculex) daumastocampa Dyar \& Knab, las cuales se colectaron en las axilas de Tillandsia biflora (Bromeliaceae: Tillandsiode) (Smith 1971, Chacín 1993) en el Parque Nacional El Ávila, entre las altitudes de 2 402-2 537 m (localidad 10), en el sendero que va desde el Pico Goering (2 $440 \mathrm{~m}$ ) hasta el Pico Naiguatá (2 765 m), máxima altura en el sistema de la costa.

En el tercer orden de altitud se encuentra Wyepmyia (Zinzala) zinzala Zavortink en el Monte (Tepui) Roraima en Pantepui, edo. Bolívar (localidad 7) a $2252 \mathrm{~m}$.

Culex quinquefasciatus Say y Culex corniger Theobald, están registradas en tres localidades $(3,9,13)$ entre 2000 y $2327 \mathrm{~m}$ en el sistema central (edo. Aragua, Cordillera Central de la Costa y edo. Trujillo, Cordillera de Los Andes). Otras especies como Culex levicastilloi Lane, Culex camposi Dyar \& Knab y Runchomyia magna Theobald, han sido registradas en máximo dos localidades por encima de los $2000 \mathrm{~m}$.

\section{DISCUSIÓN}

La mosquitofauna de fitotelmata tiene una particularidad importante con respecto a su distribución altitudinal, ya que por ser muchas de estas plantas típicas de bosques húmedos y nublados de zonas altas en áreas con bajas temperaturas promedio y con pendientes pronunciadas, las especies de mosquitos recolectadas tienden a estar en límites muy por encima de la fauna de cuerpos de agua en el suelo como lagunas, charcas, etc., en un ambiente tipo "islas" y en promedio más estables en su persistencia (Frank \& Lounibos 1987).

La cordillera andina posee las localidades de mayor altitud (Fig. 1) y por ende los registros más altos en mosquitos (Tabla 1, Fig. 1), tanto para mosquitos en fítotelmata así como para especies que utilizan otro tipo de criaderos. En fítotelmata, especies del subgénero Kerteszia de Anopheles, un grupo Neotropical de 12 especies, 11 de ellas presentes en Venezuela (Zavortink 1973, Navarro 1996b) que explotan bromelias como criadero larval principal son las especies con registros más altos, Anopheles pholidotus en el Páramos de Zumbador (Táchira) a $2680 \mathrm{~m}$ en primera instancia y otras tres especies de Kerteszia de un grupo que denominamos Grupo "boliviensis" en los Andes (Prado 2003): A. gonzalezrinconesi, A. rollai y A. boliviensis recolectadas en el Parque Nacional Dinira a 2232 m (Prado 2003, Navarro et al. 2007), mientras que Aedes euiris, ha sido referida con la altura máxima absoluta registrada para Venezuela en el Páramo Batallón y La Negra a 3133 m (Anduze 1944, Sutil 1980) y capturada como adulto.

Estas especies pueden ser indicadoras importantes para el monitoreo y conservación de la vegetación y de las áreas protegidas andinas de alta montaña, ya que por desarrollarse en las plantas epífitas adosadas a las ramas y troncos de los árboles de estas zonas húmedas y nubladas, han sufrido una disminución drástica en su abundancia, que podría indicar daños inminentes en el ecosistema de montaña andina, incluso en aquellos puntos de mayor altitud.

En el Parque Nacional El Ávila, Cordillera de la Costa, entre las altitudes de 2 402-2 537 m, en el sendero hacia el Pico Naiguatá (2 765 $\mathrm{m})$, la vegetación se encuentra en una transición de bosque nublado con vegetación baja a una vegetación alta de subpáramo en el camino final hasta el Pico Naiguatá (Steyermark 
\& Huber 1978, Meier 1998), zona en la cual la flora fitotelmata es inexistente. Por ende, la altitud máxima de la fauna de mosquitos en fitotelmata está limitada por la presencia de sus plantas hospedadoras. Entre estos dos puntos, el registro de dos especies: Wyeomyia bicornis y Culex daumastocampa (Lane 1953, Navarro \& Liria 2007) en las axilas de Tillandsia biflora, representan los registros de mayor altitud en la Cordillera de la Costa y de la zona norte de Venezuela, registro que solo podría ser superado en esta cordillera, por especies de criaderos en charcas u otro cuerpo de agua en la zona de subpáramo, cuya pendiente, drenaje y bajas temperaturas lo permitan (máx. $25^{\circ} \mathrm{C}$, $\min .0^{\circ} \mathrm{C}$ ).

Las especies $C$. daumastocampa y $W$. bicornis representan entonces, dos especies con amplio nicho en su distribución altitudinal y tipo de criaderos por estar registradas, la primera, en localidades como Panaquire edo. Miranda (en 3 especies de Araceae, 3 especies de Bromeliaceae, Machado-Allison et al. 1986) a $150 \mathrm{~m}$ de altitud y hasta los $2480-2550 \mathrm{~m}$ en el sendero a Pico Naiguatá, PN El Ávila, siendo así registrada su presencia en: Miranda, Aragua y Falcón (Sutil 1980). En el caso de W. bicornis, cuya localidad tipo es Rancho Grande, PN Henri Pittier, edo. Aragua, (>1000 m) ha sido registrada desde Ocumare de la Costa $(800 \mathrm{~m})$ hasta 2 480-2 $550 \mathrm{~m}$ y distribuida en Aragua, Barinas, Bolívar, Carabobo, Miranda, Táchira y Mérida, (Heinemann \& Belkin 1978, Sutil 1980, Navarro \& Liria 2007).

La región del Macizo Guayanés, no obstante posee varias localidades con gran altitud, es una región poco muestreada debido a las dificultades logísticas de acceso a las cimas de Tepuis en los estados Bolívar y Amazonas. En esta región se ha recolectado por encima de $2000 \mathrm{~m}$ a Wyeomyia zinzala en Roraima a una altura de 2 $252 \mathrm{~m}$ en Heliamphora nutans (Sarraceniaceae) (Barrera et al. 1989). Localidades superiores a los $2000 \mathrm{~m}$, como las del Cerro Marahuaca (2 $900 \mathrm{~m}$ ) han sido muestreadas sin éxito no así para especies de mosquitos en alturas por debajo de esa cota, como en Auyantepui, Yutaje y Guanay (Navarro et al. 2007).
Se puede concluir que la mayoría de las especies de mosquitos con un límite altitudinal elevado están presentes en la región con mayor abundancia de localidades como la región Andina (77\%), seguido de la Cordillera Central (15.3\%) y luego por el Macizo Guayanés, que tiene más localidades con alturas mayores a $2000 \mathrm{~m}$ y cuyo número de registros está subestimado por ser una localidad de difícil acceso logístico y legal. En el mismo orden, las especies que se desarrollan en fitotelmata, por estar asociados a estos ambientes tipo "islas", de gran estabilidad y permanencia (Ej. Bromelias) incluso en zonas de pendientes pronunciadas (que dificultan la existencia de criaderos en el suelo), representan el $60 \%$ de las especies con los límites de mayor altitud en las zonas montañosas de Venezuela.

También en la Tabla 1 y Fig. 1 se puede observar, registros interesantes como Culex quinquefasciatus y Culex corniger, especies asociadas al ámbito humano y cuyas larvas se crían principalmente en recipientes antropogénicos/artificiales (floreros, cauchos abandonados, bebederos de animales, canales de drenaje, etc.). Estas especies se encuentran en tres de las trece localidades $(3,9,13)$, lo que parece indicar que su límite superior de distribución altitudinal está evidentemente influenciado por la ocupación humana en localidades de gran altitud. Epidemiológicamente, estos registros de máxima distribución altitudinal, delimitaría así la posibilidad y capacidad teórica de cada una de estas especies de transmitir patógenos asociados a hospedadores susceptibles y causar problemas asociados a salud pública y veterinaria; en este sentido, Plasmodium, patógeno causante de malaria, podría ser trasmitido por las especies de Anopheles (Kerteszia) hasta una altura de $2680 \mathrm{~m}$ en el estado Táchira por medio de $A$. pholidotus, causando así la llamada "malaria de altura". Por otra parte, la especie urbana/suburbana/rural C. quinquefasciatus de hábito nocturno y marcada antropofília, podría ser potencial transmisor de patógenos como filarias, virus del Nilo occidental, recién introducido en Venezuela (Bosch et al. 2007), o encefalítis de San Luis, en los cuales ha sido 
involucrado como vector competente (Paily et al. 2006, Vanlandingham et al. 2008, Reisen et al. 2008) hasta localidades ubicadas a 2327 $\mathrm{m}$ como en Miranda, edo. Trujillo (Tabla 1 y Fig. 1).

Por el contrario, Aedes aegypti no está registrado por encima de los $2000 \mathrm{~m}$, lo que proporcionaría una barrera teórica a la transmisión de Dengue en el país. No obstante, la presencia y abundancia de esta especie en todos los estados del país, los cambios climáticos, deforestación, cambios en el uso de la tierra, ocupación humana en lugares previamente boscosos, nos hace suponer que su amplia distribución y su capacidad de colonización, pudieran aumentar su registro altitudinal, hecho que deberá corroborarse en el futuro inmediato. En Colombia Suárez \& Nelson (1981) reportaron esta especie hasta $2200 \mathrm{~m}$.

Finalmente, el mapa de ubicación de estas localidades con un perfil de alturas asociado (Fig. 1), muestra gráficamente las mayores alturas en Los Andes con respecto a la Cordillera de la Costa y Pantepui, así mismo se observa una amplia zona de llanos entre las localidades de Pantepui y las localidades de los sistemas de los Andes y de la Costa. Esta visualización está acorde con las áreas biogeográficas señaladas para mosquitos en Navarro et al. (2007) quienes señalan que existe una conexión histórica de la fauna entre Los Andes y la Cordillera Central, mientras que la fauna de Pantepui es distintiva de las anteriores. El "gap" o vacío de zonas altas en la zona baja de llanos muestra una barrera natural infranqueable entre las especies de zonas altas de Pantepui y las cordilleras promoviendo un factor adicional de aislamiento y endemismo en las especies de alta montaña en Venezuela.

\section{AGRADECIMENTOS}

A Inparques por los permisos de recolección (Parques Nacionales-Proyecto Agenda Biodiversidad 2001-2005, PN El Ávila 2009), Ministerio del Ambiente. A Mónica Prado, Nelson Moncada (muestreos en Los Andes), Ángel Rodríguez y Loriana Quintero (muestreo del PN
El Ávila). Gregorio Sanchez (Director de Investigación del IAESP, Instituto de Altos Estudios “Arnoldo Gabaldón"), Jesús Berti y Hortensia Frontado, Director y encargada respectivamente, del Museo "Pablo Cova-García" del IAESP (anteriormente DERM) por permitir el acceso a las colecciones. Financiamiento FONACIT Agenda Biodiversidad UCV-2000001339 a JCN y FONACIT LAB-2000001593: Grupo CNRA a J. Clavijo y JCN.

\section{RESUMEN}

Los mosquitos (Diptera: Culicidae) son insectos holometábolos con estadios inmaduros acuáticos que utilizan una amplia variedad de hábitats larvales, desde cuerpos de agua en el suelo hasta Fitotelmata (depósitos de agua en las plantas) y depósitos artificiales. La disponibilidad de sitios de reproducción a menudo determina el límite superior del ámbito de los mosquitos. Nosotros construimos una base de datos de 9607 registros, 432 localidades, 19 géneros y 254 especies. La coordillera Andina posee el $77 \%$ de los registros con mayor altitud incluyendo Aedes euris con un registro a $3300 \mathrm{~m}$, seguido por tres especies de Anopheles -subgénero Kerteszia- con una altitud máxima de $2680 \mathrm{~m}$. Wyeomyia bicornis y Culex daumastocampa a $2550 \mathrm{~m}$ fueron los registros de mayor altitud en la cordillera CosteraCentral, mientras que el record más alto en Pantepui fue Wyeomyia zinzala a $2252 \mathrm{~m}$. El 60\% de los registros de máxima altitud están representados por especies asociadas con fitotelmata (Bromeliaceae y Sarraceniaceae). Los límites superiores de Culex quinquefasciatus y Anopheles (Kerteszia) podría representar el límite teórico para la transmisión de filariasis o arbovirus, por Culex y malaria por Anopheles (Kerteszia) en Venezuela. Del mismo modo, un vector del dengue, Aedes aegypti, no ha sido registrado por encima de $2000 \mathrm{~m}$.

Palabras clave: Aedes, Anopheles, Bromeliaceae, Culex, Diptera, diversidad, Heliamphora, Wyeomyia.

\section{REFERENCIAS}

Anduze, P. 1944. Aedes (Ochlerotatus) euiris Dyar. Bol. Entomol. Venez. 3: 161-163.

Barrera, R. \& S. Villalba. 1994. Sucesión de especies de mosquitos (Diptera: Culicidae) en espatas de palma (Euterpe sp.) en un bosque nublado tropical. Acta Biol. Venez. 15: 25-37.

Barrera, R., D. Fish \& C.E. Machado-Allison. 1989. Ecological patterns of aquatic insects communities in two 
Heliamphora pitcher-plant species of the Venezuelan highlands. Ecotropicos 2: 31-44.

Bosch, I., F. Herrera, J.C. Navarro, M. Lentino, A. Dupuis, J. Maffei, M. Jones, E. Fernandez, N. Perez, N. Komar, J. Pérez, A.E. Guimaraes, R. Barrera, N. Valero, J. Ruiz, J. Rivero, I. Pérez, M. Méndez, J. Martinez, G. Velásquez, I. Matheus, G. Comach, A. Spielman \& L. Kramer. 2007. West Nile virus, Venezuela. Emerg. Infect. Dis. 13: 651-653.

Cárdenas, A.L. 1965. Geografía Física de Venezuela, Talleres Gráficos Universitarios, Mérida, Venezuela.

Chacín, I. 1993. Distribución geográfica de la familia Bromeliaceae en Venezuela. Trabajo Especial de Grado, Escuela de Biología, Universidad Central de Venezuela. Caracas, Venezuela.

Cova-García, P. 1951. Distribución Geográfica y Datos Bionómicos de los Anofelinos de Venezuela. Publicaciones de la División de Malariología. Número 10. Ministerio de Sanidad y Asistencia Social Caracas, Venezuela.

Cova-Garcia, P., E. Sutil, J.A. Rausseo. 1966. Mosquitos de Venezuela (Tomo I). Publicaciones del Ministerio de Sanidad y Asistencia Social, Caracas, Venezuela.

Del Ventura, F. 2008. Patrones de distribución, análisis geoespacial y determinación de áreas de endemismo de los mosquitos (Diptera: Culicidae) de Venezuela. Trabajo Especial de Grado, Escuela de Biología, Universidad Central de Venezuela. Caracas, Venezuela.

Eguía, C. 1991. Venezuela. I. Geografía.1. Medio Físico. Gran Enciclopedia Rialp: Humanidades y Ciencia. Caracas, Venezuela (también disponible en línea: http://www.canalsocial.net/GER/ficha_GER. asp?id=7379\&cat=geografia).

Fish, D. 1983. Phytotelmata: Flora and Fauna, p.1-25, In J.H. Frank, L.P. Lounibos (eds.). Phytotelmata: terrestrial plants as hosts for aquatic insect communities. Plexus, Nueva Jersey, EEUU.

Frank, J.H. 1983. Bromeliad phytotelmata and their biota, especially mosquitoes, p.101-128. In J.H. Frank, L.P Lounibos (eds.). Phytotelmata: terrestrial plants as hosts for aquatic insect communities. Plexus, Nueva Jersey, EEUU.

Frank, J.H. \& L.P. Lounibos.1987. Phytotelmata: swamps or islands? Fla. Entomol. 70: 14-20.

González de Juana, C. 1938. Contribución al estudio de la cuenca sedimentaria Zulia-Falcón. Bol. Geol. y Min. II: 2-4.
Guimaraes, J.H. 1997. Systematic Database of Diptera of the Americas South of the United States, Family Culicidae. São Pablo. Fundação de Amparo a Pesquisa do Estado de São Pablo. Pleide. Brasil.

Harbach, R.E. \& J.C. Navarro. 1996. A new species of Anopheles subgenus Kerteszia (Diptera: Culicidae) from Venezuela. Entomol. Scand. 27: 207-216.

Heinemann, S.J. \& J.N. Belkin. 1978. Collection records of the project "Mosquitoes of Middle America". 11. Venezuela (VZ); Guianas French Guiana (FC, FCC), Guyana (GUY), Surinam (SUR). Mosq. Syst. 10: 365-459.

Knight, K.L. \& A. Stone. 1977. A Catalog of the Mosquitoes of the World (Diptera: Culicidae). Vol. VI. Thomas Say Found, Entomological Society of America, EEUU.

Lane, J. 1953. Neotropical Culicidae. University of Sao Paulo, Sao Paulo, Brazil.

Liria, J. \& J.C. Navarro 2003. Morphology of the larval mandibles and maxillae in the subgenus Carrollia of Culex (Diptera: Culicidae). Zootaxa 186: 1-8.

Lounibos, L.P. \& C.E. Machado-Allison. 1983. Oviposition and egg brooding by the mosquito Trichoprosopon digitatum in cacao husks. Ecol. Entomol. 8: 475-478.

Machado-Allison, C.E., R. Barrera, J.H. Frank, L. Delgado \& C. Gomez-Cova. 1985. Mosquito communities in Venezuela phytotelmata, p. 79-93. In L.P. Lounibos, J.R. Rey, J.H. Frank (eds.). Ecology of mosquitoes. Vero Beach, Florida, EEUU.

Machado-Allison, C.E., R. Barrera, L. Delgado, C. GomezCova \& J.C. Navarro. 1986. Mosquitos (Diptera: Culicidae) de los Fitotelmata de Panaquire, Venezuela. Acta Biol. Venez. 12: 1-12.

Maguire, B. 1971. Phytothelmata biota and community structure determination in plant-held waters. Ann. Rev. Ecol. Syst. 2: 439-464.

Meier, W. 1998. Flora und Vegetation des Ávila-Nationalparks (Venezuela/Kustenkordillere) unter besonderer Berucksichtigung der Nebelwaldstufe. Dissertationes Botanicae, Tomo 296. Borntraerger Verlagsbuchhandlung-Berlin-Stuttgart. Alemania.

Navarro, J.C. 1996a. Toxorhynchites haemorrhoidalis superbus (Dyar \& Knab 1906), nuevo registro para la fauna de Culicidae de Venezuela. Acta Biol. Venez. 16: 63-67. 
Navarro, J.C. 1996b. Nueva clave para la identificación de larvas de los Anophelini de Venezuela, con la actualización de la taxonomía del grupo. Bol. Malariol. San. Amb. Vol. XXXVI: 25-43.

Navarro, J.C. 1998. Fauna de mosquitos del P.N. Cerro Copey, nuevos registros para Nueva Esparta. Bol. Entomol. Venez. 3: 187-194.

Navarro, J.C. \& J. Liria. 2000. Phylogenetic relationships among eighteen Neotropical Culicini species. J. Am. Mosq. Control Assoc. 16: 75-85.

Navarro, J.C. \& J. Liria. 2007. Wyeomyia trujilloi Pulido y Sutil 1981, nuevo sinonimo de Wy. bicornis (Root 1928) (Culicidae: Sabethini), con redescripción de la pupa y parte de la larva. Bol. Malariol. San. Amb. 47: 35-48.

Navarro, J.C., R.J. Bastidas \& Y. Zavala. 1994. Fauna de Mosquitos (Diptera: Culicidae) del Estado Falcón, Venezuela. I. Nuevos Registros y Listado General de Especies. Acta Cient. Venez. 45: 315-324.

Navarro, J.C., J. Ingunza, Z. Fernández \& R. Barrera 1995. Mosquitoes and bromeliads: species-specific selectivity patterns on the northern coast and southern Guiana Shields in Venezuela. J. Am. Mosq. Control Assoc. 11: 345-346.

Navarro, JC., J. Liria, H. Piñango \& R. Barrera. 2007. Biogeographic area relationships in Venezuela: A Parsimony analysis of Culicidae-Phytotelmata relationships distributions in National Parks. Zootaxa 1547: 1-19.

Paily, K.P., S.L. Hoti, \& K. Balaraman 2006. Development of lymphatic filarial parasite Wuchereria bancrof$t i$ (Spirurida: Onchocercidae) in mosquito species (Diptera: Culicidae) fed artificially on microfilaremic blood. J Med Entomol. 43: 1222-6.

Prado, M. 2003. Caracterización morfológica y molecular de tres especies del subgénero Kerteszia de Anopheles (Diptera: Culicidae) en la región andina venezolana. Trabajo Especial de Grado, Escuela de Biología, Universidad Central de Venezuela. Caracas, Venezuela.

Reisen, W.K., H.D. Lothrop, S.S. Wheeler, M. Kennsington, A. Gutierrez, Y. Fang \& S.B. Lothrop. 2008 Persistent West Nile virus transmission and the apparent displacement St. Louis encephalitis virus in southeastern California, 2003-2006. J. Med. Entomol. 45: 494-508.

Rubio-Palis, Y. \& R.H. Zimmermann. 1997. Ecoregional classification of malaria vectors in the neotropics. J. Med. Entomol. 34: 499-510.

Schubert, C. 1982. Glacial geology of Paramo Batallon, State of Táchira, Venezuela. Act. Cient. Venez. 33 66-71.

Seifert, R.P. 1980. Neotropical Heliconia insect communities. Quat. Rev. Biol. 57: 1-28.

Seifert, R.P. \& R. Barrera. 1981. Cohort studies on mosquito (Diptera: Culicidae) larvae living in the water-filled floral bracts of Heliconia caribaea (Zingiberales, Musaceae). Ecol. Entomol. 6: 191-197.

Smith, L.B. 1971. Bromeliaceae. Vol. XII. Primera Parte. Instituto Botánico, Dirección de Recursos Naturales Renovables. Ministerio de Agricultura y Cría, Caracas, Venezuela.

Steyermark, J. \& O. Huber. 1978. Flora del Avila: flora y vegetación de las montañas del Ávila, de la Silla y del Naiguatá. Sociedad Venezolana de Ciencias Naturales. Ministerio del Ambiente y de los Recursos Naturales Renovables. Caracas, Venezuela.

Suárez, M.F. \& M.J. Nelson. 1981. Registro de Altitud del Aedes aegypti en Colombia. Biomedica 1: 225.

Sutil, O.E. 1980. Enumeración histórica y geográfica de las especies de Culicidae de Venezuela ordenadas según su taxonomía. Bol. Dir. Malariol. San. Amb. 20: 1-32.

Vanlandingham, D.L, C.E. McGee, K.A. Klingler, S.E. Galbraith, A.D. Barrett \& S. Higgs. 2008. Comparison of oral infectious dose of West Nile virus isolates representing three distinct genotypes in Culex quinquefasciatus. Am. J. Trop. Med. Hyg. 79: 951-4.

Zavortink, T.J. 1973. Mosquito Studies (Diptera, Culicidae). XXIX. A review of the subgenus Kerteszia of Anopheles. Contrib. Am. Entomol. Inst. (Ann Arbor) 9: 1-54.

\section{REFERENCIA DE INTERNET}

WRBU. 2009. Walter Reed Biosystematics, Systematic Catalog of Culicidae. Washington DC, EEUU (Consultado: julio 16 2009, http://www.mosquitocatalog.org). 